\title{
Spin relaxation in submonolayer and monolayer InAs structures grown in a GaAs matrix
}

\author{
Chunlei Yang, ${ }^{1,2}$ Xiaodong Cui, ${ }^{1, *}$ Shun-Qing Shen, ${ }^{1}$ Zhongying Xu ${ }^{3}$ and Weikun $\mathrm{Ge}^{2}$ \\ ${ }^{1}$ Department of Physics, The University of Hong Kong, Hong Kong, China \\ ${ }^{2}$ School of Physics and Engineering, Sun Yat-Sen University, 510275 Guangzhou, China \\ ${ }^{3}$ State Key Laboratory for Superlattices and Microstructures, Institute of Semiconductors, Chinese Academy of Sciences, \\ 100083 Beijing, China
}

(Received 12 January 2009; revised manuscript received 13 June 2009; published 22 July 2009)

\begin{abstract}
Electron-spin dynamics in InAs/GaAs heterostructures consisting of a single layer of InAs (1/3-1 monolayer) embedded in (001) and (311)A GaAs matrix was studied by means of time-resolved Kerr rotation spectroscopy. The spin-relaxation time of the submonolayer InAs samples is significantly enhanced, compared with that of the monolayer InAs sample. The electron-spin-relaxation time and the effective $g$ factor in submonolayer samples were found to be strongly dependent on the photogenerated carrier density. The contribution from both the D'yakonov-Perel' mechanism and Bir-Aronov-Pikus mechanism are discussed to interpret the temperature dependence of spin decoherence at various carrier densities.
\end{abstract}

DOI: 10.1103/PhysRevB.80.035313

\section{INTRODUCTION}

One of the pioneering approaches toward prospective spintronic devices is to manipulate electron spins by utilizing spin-orbit coupling in nonmagnetic semiconductors, particularly in low-dimensional III-V semiconductor heterostructures (quantum wells, wires, and dots) owing to their great flexibility in manipulating spin properties of the electronic states. ${ }^{1}$ Unlike electron charge, electron spin is not conserved and generally relaxes to unpolarized states in solids. There exist competing spin-relaxation channels: spin flip through electron-impurity scattering, known as Elliott and Yafet mechanism, ${ }^{2}$ spin flip through electron-hole exchange scattering known as Bir-Aranov-Pikus (BAP) mechanism, ${ }^{3}$ and spin flip through spin-orbit coupling known as D'yakonovPerel' (DP) mechanism. ${ }^{4}$ The relative importance of these mechanisms is strongly dependent on sample structure, temperature, and carrier concentration.

InAs/GaAs heterostructures as one of the potential spintronic building blocks have received intensive attention during the last decade. InAs submonolayer in a GaAs matrix is a very unique system which shows different optical properties from those of InAs single layer and InAs quantum dots. $^{5-7}$ The lateral size of the islands formed in InAs submonolayers can be easily and continuously tuned in a large range. Most importantly, there is a narrow size distribution for these islands as revealed by their sharp photoluminescence (PL) spectra. ${ }^{8}$ It is of advantage to utilize spin states of submonolayer structures for the sake of almost unified electronic states which are hard to achieve for self-assembled quantum dots due to wide size distribution. Nevertheless, there are few experiments addressing the spin dynamics in ultrathin InAs layers.

In this paper, we studied the spin relaxation in InAs/GaAs heterostructures consisting of a single layer of InAs with effective thickness of $1 / 3,1 / 2$, and 1 monolayer by timeresolved Kerr rotation (KR) spectroscopy. The spinrelaxation time was found to increase with the decrease in InAs coverage. The contribution from both DP mechanism and BAP mechanism were discussed to interpret the temperature dependence of spin decoherence at various carrier densities.
PACS number(s): 73.21.Fg, 72.25.Rb, 78.47.jc

\section{EXPERIMENT}

Ultrathin InAs layer is a kind of InAs/GaAs heterostructure in which a single InAs layer is embedded in a GaAs matrix. The samples were grown by elemental source molecular-beam epitaxy on (001) and (311)A-oriented GaAs semi-insulating substrates. The structures consist of a GaAs buffer layer, two cladding layers, and an InAs layer. Each cladding layer is composed of 40 period GaAs $/ \mathrm{Al}_{0.4} \mathrm{Ga}_{0.6} \mathrm{As}$ superlattices (SL) and a GaAs layer. The use of GaAs/ AlGaAs SL improves sample quality by preventing surface recombination and trapping defects. The single InAs layer with various effective thicknesses is sandwiched between the cladding layers. To study the lateral size effect on the spinrelaxation process, three samples on (001) substrates with $1 / 3,1 / 2$, and 1 monolayer (ML) InAs, respectively, and one sample on (311)A substrate with 1 ML InAs were prepared. All the samples are nominally undoped. The details of the growth and the optical characterizations of the samples could be found elsewhere. ${ }^{8}$ The 1 ML InAs layer on (001) surface can be treated as an ideal two-dimensional (2D) system. Instead, due to the intrinsic surface corrugation, ${ }^{9}$ the $1 \mathrm{ML}$ InAs layer on (311)A surface forms wirelike or disklike microstructures on the GaAs steps and facets. While for the $1 / 2$ and 1/3 ML samples on (001) surface, InAs is found to be organized as disklike islands elongated along [011] direction with lateral size of tens of nanometers due to the fractional surface coverage..$^{10}$ The four samples, with the same thickness but different lateral sizes, provide an excellent system to demonstrate the suppression of spin-relaxation process by lateral size constriction down to tens of nanometer, without bringing significant spin scattering by the edge defects as observed in narrow channel devices fabricated by etching process. ${ }^{11}$

To study the coherent spin dynamics, an optical pumpprobe spectroscopy technique called time-resolved KR spectroscopy is used. A circularly polarized pump pulse generates a well-defined carrier spin population and the KR angle of a linearly polarized probe light is detected by a balanced optical bridge. The time delayed probe pulse reflects the time evolution of the projection of the electron-spin states, which 


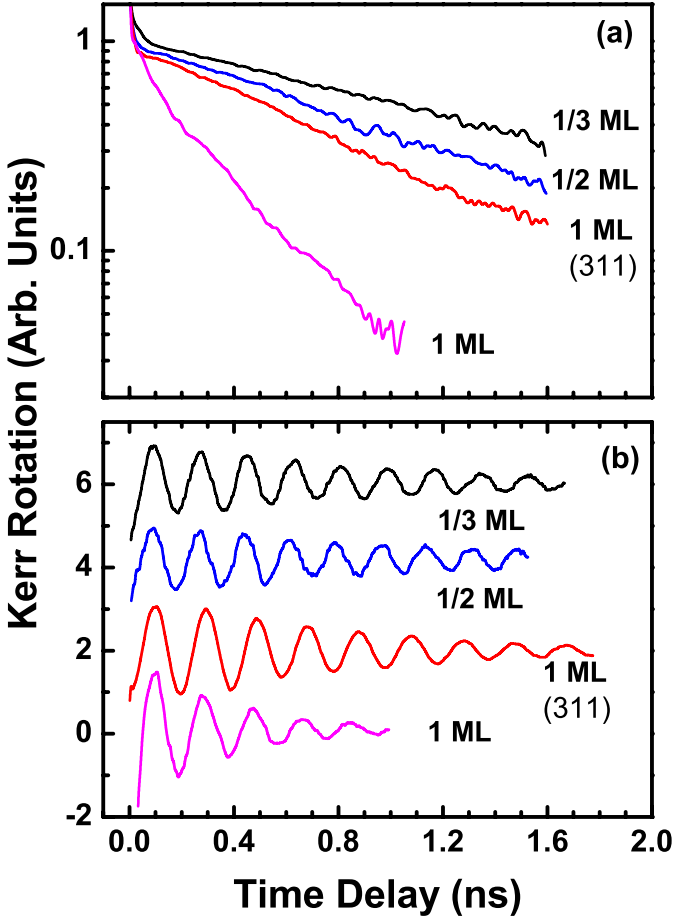

FIG. 1. (Color online) Kerr rotation at (a) $B=0 \mathrm{~T}$ and (b) $B$ $=0.82 \mathrm{~T}$ for samples with InAs thickness of $1 / 3,1 / 2$, and $1 \mathrm{ML}$, respectively. The temperature is at $77 \mathrm{~K}$. The data of the sample on (311) substrate is also shown. The pumping density is about 5 $\times 10^{16} / \mathrm{cm}^{3}$

precesses perpendicularly to the transverse external magnetic field. Both pump and probe pulses are obtained from a tunable mode-locked Ti:Sapphire laser with a pulse width about $150 \mathrm{fs}$ and a repetition rate of $80 \mathrm{MHz}$. The pump beam size is about $30 \mathrm{~mm}$ in focus and the size of the probe beam is tuned to be a little bit smaller than that. The typical excitation powers are $0.5-10 \mathrm{~mW}$ for the pump and $0.5 \mathrm{~mW}$ for the probe beams. To get an excellent signal-to-noise ratio, a double lock-in detection technique is employed with the amplitude modulation of the probe beam at $115 \mathrm{~Hz}$ with an optical chopper and the polarization modulation of the pump beam at $50 \mathrm{KHz}$ with a photoelastic modulator. The measurements were carried out in a magneto-optical cryostat in Voigt geometry with a tunable photon energy of $1.45-1.5 \mathrm{eV}$.

\section{RESULTS AND DISCUSSION}

Figure 1(a) shows the KR signals at zero external field for samples with InAs thickness of $1 / 3,1 / 2$, and $1 \mathrm{ML}$, respectively. The temperature was $77 \mathrm{~K}$ and the pumping density was kept at low level with pumping power of $0.5 \mathrm{~mW}$ and the carrier density was estimated to be about $5 \times 10^{16} / \mathrm{cm}^{3}$. These data depict that there is a fast decay of the spin polarization during the very first $10 \mathrm{ps}$ after excitation and followed by a long-lifetime simple exponential decay process. The fast decay originates from the loss of hole spin polarization since the hole spin lifetime is short due to strong valence-band mixing and $k$-dependent spin splitting. ${ }^{12}$ The evolution of the KR angle thereafter can be described by a single exponential decay $\theta_{k}=A_{0} \exp \left(-\frac{t}{\tau_{s}}\right)$ for all the samples, where $A_{0}$ is proportional to the initial amplitude of the electron-spin polarization, $t$ is the time delay between the circularly polarized pump and the linearly polarized probe pulse, and $T_{2}^{*}$ is the electron-spin-relaxation time. The independent evolution of the electron- and hole-spin polarization indicates that we do not need to take the exciton spin as a constituent as previously studied using time-resolved photoluminescence at those low temperatures. ${ }^{13}$ The spinrelaxation time for the $1 / 3,1 / 2$, and $1 \mathrm{ML}$ samples on (001) surface are extracted to be 1500,984 , and $380 \mathrm{ps,} \mathrm{respec-}$ tively. The spin-relaxation time for the $1 \mathrm{ML}$ sample on (311)A surface is determined to be $860 \mathrm{ps}$. These data clearly evidence that the electron-spin lifetime is significantly increased as the lateral size is gradually reduced, of which the mechanism will be discussed later.

Figure 1(b) exhibits the KR data at in-plane external magnetic field $B=0.82 \mathrm{~T}$ for the four samples. It shows a clear spin oscillation under the transverse external magnetic field, which can be well described by $S_{0} \exp \left(-t / T_{2}^{*}\right) \cos \left(g^{*} \mu_{B} B t / \hbar\right)$, where $S_{0}$ is the initial amplitude, $T_{2}^{*}$ is the inhomogeneous transverse electron-spin lifetime, $g^{*}$ stands for the effective electron $g$ factor, $\mu_{B}$ and $\hbar$ are the Bohr magneton and Planck constant, respectively. $T_{2}^{*}$, s extracted from the spin oscillation at $0.82 \mathrm{~T}$ are found to be almost the same as the spin-relaxation times measured at zero field. The magnitude of the extracted electron $g$ factor for the four samples falls in the narrow range of $0.46-0.48$ which is remarkably different from that of bulk InAs of about 15. The discrepancy of the electron $g^{*}$ factor in these ultrathin quantum wells from the bulk value is attributed to the penetration of carrier wave function into the barriers, quantum confinement energy, ${ }^{14}$ strain, ${ }^{15}$ etc.

The lack of inversion symmetry in such III-V compounds of zinc-blende structures such as GaAs and InAs results in spin splitting of the conduction band via spin-orbit coupling. Spin-orbit coupling contributes a momentum-dependent effective magnetic field. This is the driving force for spin relaxation in DP theory. The mechanism of the electron-spin decoherence occurs via the spin precession of carriers with finite crystal momentum $k$ caused by the effective $k$-dependent magnetic field in an inversion-asymmetric material. Since spin polarization changes during precession between scatterings, scattering acts against spin relaxation and accordingly the spin lifetime is inversely proportional to the momentum scattering time $\tau_{p}$ (namely, $\tau_{s}-\tau_{p}^{-1}$ ). A signature of this mechanism is that in the motional narrowing regime where spin coherence time greatly exceeds momentum scattering time $\tau_{p}$, cleaner samples are expected to have shorter spin coherence time. ${ }^{16}$ It is naturally expected that scattering by boundaries and deformation potentials will decrease $\tau_{p}$ in InAs submonolayer samples, where the submonolayer exists in form of disks with lateral size of tens of nanometer and consequently boundaries and deformation potentials are enhanced with the decreased coverage. The decreased $\tau_{p}$ in InAs submonolayer will increase the spin-relaxation time. On the other hand, the PL data ${ }^{8}$ shows that inhomogeneous broadening increases with the InAs coverage. It is known that inhomogeneous broadening in the DP picture also will 
$\begin{array}{llllllllll}0.0 & 0.4 & 0.8 & 1.2 & 1.6 & 0.0 & 0.4 & 0.8 & 1.2 & 1.6\end{array}$

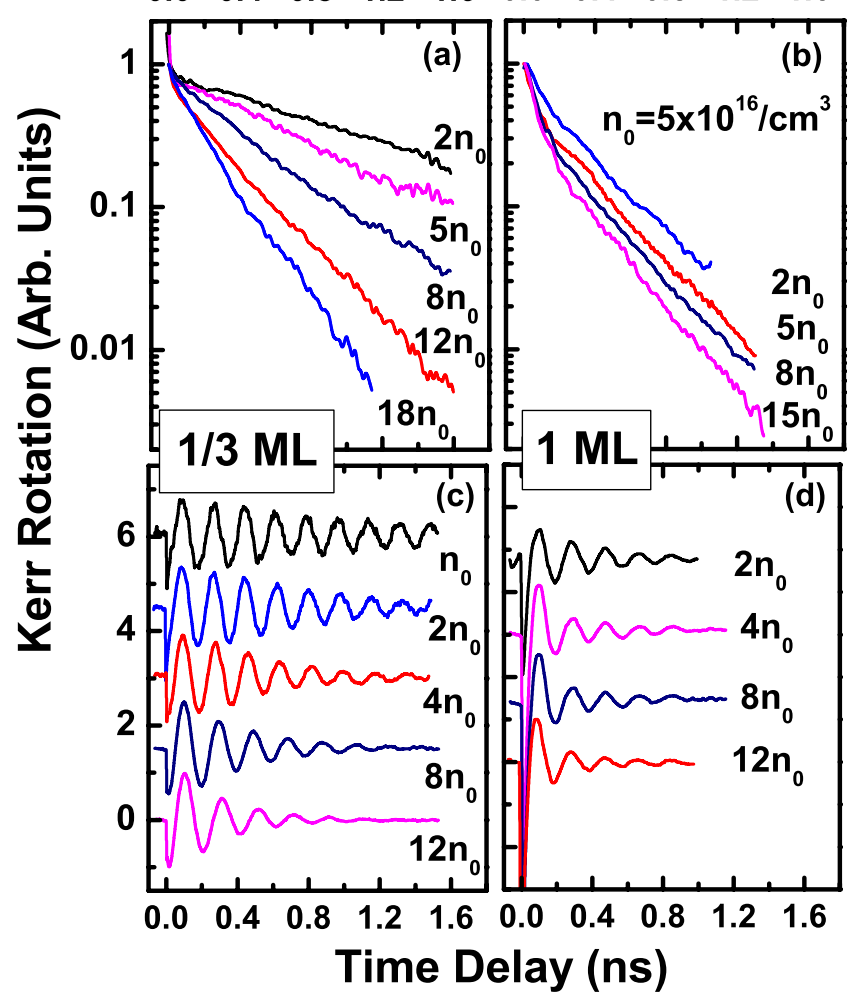

FIG. 2. (Color online) Kerr rotation at $B=0 \mathrm{~T}$ for (a) $1 / 3 \mathrm{ML}$ and (b) 1 ML InAs samples, respectively, with various pumping density as indicated. (c) and (d) are the pumping-density-dependent Kerr rotation of $1 / 3$ and $1 \mathrm{ML}$ InAs samples at $B=0.82 \mathrm{~T}$, respectively. The temperature is $77 \mathrm{~K}$.

induce spin decoherence ${ }^{17}$ due to the dephasing process of the spin ensemble. The different inhomogeneous broadening also benefits the increase in spin lifetime in submonolayers by DP mechanism.

Another important feature we found is that the spin lifetime in the submonolayer InAs samples is strongly dependent on carrier density. The KR data for $1 / 3$ and 1 ML InAs (001) samples at $B=0$ and $0.82 \mathrm{~T}$ are shown in Figs. 2(a)-2(d), respectively, with various pumping density as indicated. In both zero field and transverse external magnetic field cases, one can clearly see that the higher pumping intensity, the faster decay of spin polarization in the $1 / 3 \mathrm{ML}$ sample. In contrast, the decay of the spin polarization in the $1 \mathrm{ML}$ sample is not that sensitive to the pumping density.

To get more insights into the spin-relaxation mechanisms, we plot the derived electron-spin decoherence time $T_{2}^{*}$ and the effective $g$ factor as a function of carrier density for the three samples on (001) substrates. In Fig. 3(a), we can find that $T_{2}^{*}$ in both $1 / 3$ and $1 / 2$ ML samples drop quickly as carrier density increases, especially in the low-carrier-density region; while that of the 1 ML sample displays weak dependence on carrier density. We have shown that the spin relaxation by DP mechanism in the submonolayers is suppressed due to the lateral size confinement. The BAP mechanism involving electron-hole exchange interaction may, however, be enhanced or even become dominant in these quantum disk structures because of the strong interaction between the spa-

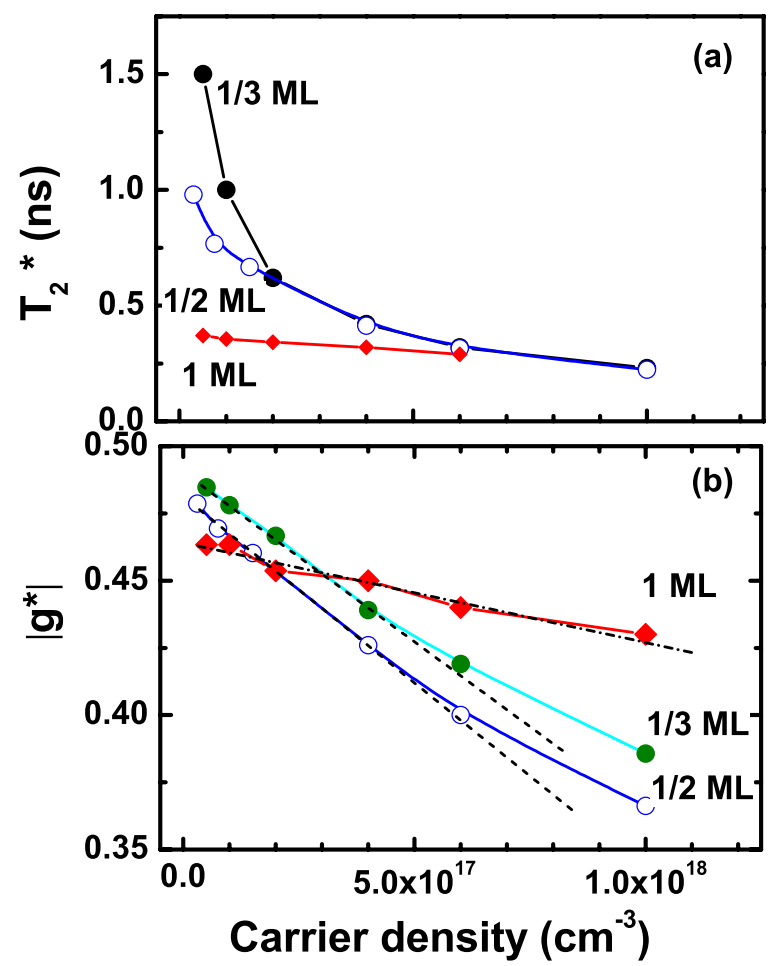

FIG. 3. (Color online) (a) The derived electron-spin decoherence time $T_{2}^{*}$ and (b) effective $g$ factor for the three samples on (100) substrates with various carrier density. The temperature is $77 \mathrm{~K}$.

tially confined photoexcited electrons and holes. The fact that $T_{2}^{*}$ decreases with increasing carrier density also agrees well with the qualitative expectation of the BAP process. As predicted by Ref. 18, however, it is questioned that the effect of the BAP mechanism at low temperature and high electron density is far exaggerated in the literature due to the neglect of the nonlinear terms in the spin-flip electron-hole exchange scattering. We will discuss the relevant importance of BAP and DP effects later using temperature-dependent Kerr rotation at various pumping densities and it shows that BAP is not the main reason for the fast decrease in the spin lifetime with the increase in pumping density.

In Fig. 3(b), we plot the carrier density dependence of $g^{*}$ for three different samples. The measured $g^{*}$ by transient Kerr rotation should correspond to that at the quasi-Fermi energy of photogenerated electrons. It has been found that within a small energy range, the effective $g$ factor can be approximated by $g^{*}=g_{0}+\beta E$, where $\beta$ denotes a constant and $E$ denotes the energy. ${ }^{19}$ The electron density of states (DOS) follows $D(E)=d n / d E$, where $n$ is the electron density. Given a measurement of $g^{*}$ as a function of electron density, the electron DOS can therefore be determined by $D(E)$ $=\beta\left(d g^{*} / d n\right)^{-1}$, where $n$ is the electron density up to the quasi-Fermi energy. In a 2D system, a linear dependence of $g^{*}$ on the electron density is expected, given that the DOS of the $2 \mathrm{D}$ system is a constant independent of energy. ${ }^{20}$ This expectation agrees well with our experimental results particularly on the $1 \mathrm{ML}$ sample, as shown in Fig. 3(b). For submonolayer samples, the slight discrepancy from linear relation is attributed to the lateral confinement in these 


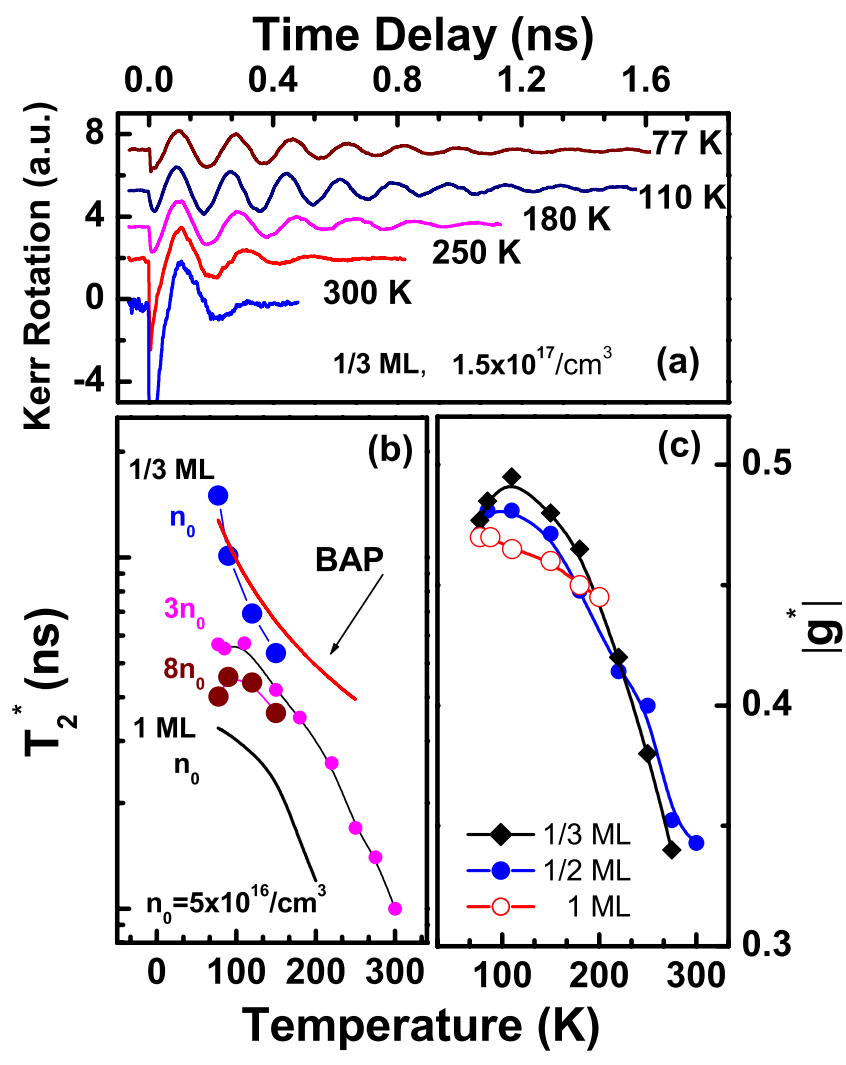

FIG. 4. (Color online) (a) Kerr rotation of the 1/3 ML InAs sample at different temperatures measured at $B=0.82 \mathrm{~T}$. The pumping density is about $1.5 \times 10^{17} / \mathrm{cm}^{3}$. The temperature dependence of the electron-spin decoherence time $T_{2}^{*}$ and the effective $g$ factor are shown in (b) and (c), respectively. The $T_{2}^{*}$ of 1/3 ML InAs sample with various pumping density at low temperature as specified is also shown for comparison.

quasi-2D systems (quantum islands-like), which will be addressed later.

To carefully identify the spin decoherence mechanism in the submonolayer samples, we have measured temperaturedependent KR with various pumping densities. Figure 4(a) shows some of the KR data of the $1 / 3 \mathrm{ML}$ InAs sample at different temperatures measured at $\mathrm{B}=0.82 \mathrm{~T}$ with pumping density about $1.5 \times 10^{17} / \mathrm{cm}^{3}$. The temperature dependence of the extracted electron-spin decoherence time $T_{2}^{\star}$ and effective $g$ factor are shown in Figs. 4(b) and 4(c), respectively. The $T_{2}^{*}$ of $1 / 3 \mathrm{ML}$ InAs sample with various pumping density as specified is also shown for comparison.

BAP mechanism predicts spin-relaxation time decreases rapidly with increased temperature at low temperatures but is less sensitive to temperature at higher temperatures. ${ }^{18}$ The experimental data shown in Fig. 4(b) indicates that only the data of $1 / 3 \mathrm{ML}$ at low temperature with low pumping intensity (such as $5 \times 10^{16} / \mathrm{cm}^{3}$ ) agrees with this dependence. The line named BAP in Fig. 4(b) is a fit of the data of the 1/3 ML sample at low temperature and low carrier density (5 $\left.\times 10^{16} / \mathrm{cm}^{3}\right)$, assuming $T^{-1}$ dependence of the BAP mechanism. ${ }^{21}$ When the pumping intensity increases [for example, $1.5 \times 10^{17} / \mathrm{cm}^{3}$ and above shown in Fig. 4(b)], the spin lifetime at low temperature shows less sensitive to temperature, obviously deviating from $T^{-1}$ dependence of the
BAP mechanism. The spin lifetime at higher carrier density $\left(4 \times 10^{17} / \mathrm{cm}^{3}\right)$ even displays a peak at around $100 \mathrm{~K}$. These observations clearly demonstrate that the BAP mechanism is not the dominant process at higher carrier density or at higher temperature. Actually, all the observations could be well described by the mechanisms involving both BAP and DP mechanism. The BAP mechanism is believed to be only dominant in submonolayer samples at low temperature with low carrier density. With the increase of temperature or carrier density, the contribution from DP mechanism grows quickly and eventually dominates the spin-relaxation process. It is the combination of BAP and DP mechanisms that makes the spin decoherence time in InAs submonolayers exhibit complicated behavior upon the change in temperature and carrier density. While for the $1 \mathrm{ML}$ sample, DP mechanism is believed to dominate at both low temperature and higher temperature.

To the authors' knowledge, the above interesting phenomena can be well understood if we take the lateral confinement in these submonolayer samples into consideration. For the submonolayer samples we studied, they are quasi-2D systems with some localization due to the lateral confinement. The confined electronic states in InAs are very close to the conduction-band minimum of GaAs barrier, which suggests strong penetration of the envelope wave function into the barrier. The carriers in neighboring islands are believed to have strong coupling and there might be large probabilities for them to tunnel between neighboring islands. This partially explains the lateral mobility of the carriers in the plane, despite the topographical islandlike structure, and makes the origin of its $2 \mathrm{D}$ nature. This has been suggested by previous observation that the carrier lifetime in InAs submonolayer samples increase linearly with temperature. ${ }^{8}$ At low temperature and low carrier density, the photogenerated carriers in submonolayer samples occupy mainly the states with minimum energy in the plane. The localization (or lateral constriction) of the carriers strongly suppresses the DP processes and makes BAP process possibly dominate the spin relaxation. Different to ideal quantum dots, the in-plane mobility (2D nature) of carriers in the submonolayer leads to 2D-like energy-momentum dispersion, which makes the $g^{*}$ factor of InAs submonolayer linearly dependent on carrier density, just like quantum well. At higher pumping density and/or at higher temperature, carriers populate at higher energy states and they move more freely in the plane. This consequently increases the spin-flip probability by DP process that is strongly related to momentum, which well explains the dramatic decrease in the spin-relaxation time in submonolayer with the increase in carrier density or temperature. Besides, although both show linear dependence at lower density in Fig. 3, the slope $(d g * / d n)$ for submonolayer samples is much larger than that for monolayer sample, which is attributed to the limited DOS for the fractional coverage and localization. At higher pumping (carrier) density, carriers tend to populate at higher states which corresponds to higher DOS. The reduced slope of $d g^{*} / d n$ at high excitation density in the submonolayer samples as shown in Fig. 3(b) further supports the scenario. The experimentally observed quenching of DP process at low temperature and dominating DP process at high temperature/high excitation in submonolayer 
can be well explained by the above analysis. Figure 4(c) depicts the temperature dependence of the $g^{*}$ factor for the three InAs samples at pump density around $1.5 \times 10^{17} / \mathrm{cm}^{3}$. The submonolayer samples exhibit a humplike dependence of the $g^{*}$ factor on temperature, which may originate from the thermal redistribution of the carriers in energy states.

In conclusion, we have experimentally studied the spinrelaxation process in InAs ultrathin layer embedded in GaAs matrix. Long spin-relaxation time in the submonolayer structures has been observed and been attributed to the suppression of DP process owing to constriction of lateral dimensions. The electron-spin-relaxation time and effective $g$ factor in submonolayer samples were found to be strongly dependent on the photon-generated carrier density. The dependence of spin relaxation on temperature has been examined and the related mechanisms have been discussed. The clear coherent spin oscillation at $300 \mathrm{~K}$ in the InAs ultrathin layer seems attractive for coherent spin manipulation at room temperature.

\section{ACKNOWLEDGMENTS}

The authors thank M. W. Wu for helpful discussions. This work was supported by Hong Kong GRF under Grant No. HKU701308P, China NSF under Grants No. 60706021, No. 10874248, and No. 60876066. *xdcui@hku.hk

${ }^{1}$ Semiconductor Spintronics and Quantum Computation, edited by D. D. Awschalom, N. Samarth, and D. Loss (Springer, Heidelberg, 2002); I. Žutić, J. Fabian, and S. Das Sarma, Rev. Mod. Phys. 76, 323 (2004); J. Fabian, A. Matos-Abiague, C. Ertler, P. Stano, and I. Zutic, Acta Phys. Slov. 57, 565 (2007); Spin Physics in Semiconductors, edited by M. I. D'yakonov (Springer, Berlin, 2008).

${ }^{2}$ Y. Yafet, Phys. Rev. 85, 478 (1952); R. J. Elliott, ibid. 96, 266 (1954).

${ }^{3}$ G. L. Bir, A. G. Aronov, and G. E. Pikus, Sov. Phys. JETP 42, 705 (1976).

${ }^{4}$ M. I. D’yakonov and V. I. Perel', Zh. Eksp. Teor. Fiz. 60, 1954 (1971).

${ }^{5}$ G. Dresselhaus, Phys. Rev. 100, 580 (1955).

${ }^{6}$ Y. A. Bychkov and E. I. Rashba, Pis'ma Zh. Eksp. Teor. Fiz. 39, 66 (1984) [JETP Lett. 39, 78 (1984)].

${ }^{7}$ Matthias Ilg, M. Isabel Alonso, Arno Lehmann, Klaus H. Ploog, and Matthias Hohenstein, J. Appl. Phys. 74, 7188 (1993).

${ }^{8}$ Z. L. Yuan, Z. Y. Xu, B. Z. Zheng, J. Z. Xu, S. S. Li, Weikun Ge, Y. Wang, J. Wang, L. L. Chang, P. D. Wang, C. M. Sotomayor Torres, and N. N. Ledentsov, Phys. Rev. B 54, 16919 (1996).

${ }^{9}$ Klaus H. Ploog, Richard Nötzel, and Matthias Ilg, J. Vac. Sci. Technol. B 11, 1675 (1993).

${ }^{10}$ V. Bressler-Hill, A. Lorke, S. Varma, P. M. Petroff, K. Pond, and
W. H. Weinberg, Phys. Rev. B 50, 8479 (1994).

${ }^{11}$ A. W. Holleitner, V. Sih, R. C. Myers, A. C. Gossard, and D. D. Awschalom, Phys. Rev. Lett. 97, 036805 (2006).

${ }^{12}$ T. C. Damen, Luis Vina, J. E. Cunningham, Jagdeep Shah, and L. J. Sham, Phys. Rev. Lett. 67, 3432 (1991).

${ }^{13}$ Zheng Sun, Z. Y. Xu, Yang Ji, B. Q. Sun, B. R. Wang, S. S. Huang, and H. Q. Ni, Appl. Phys. Lett. 90, 071907 (2007).

${ }^{14}$ M. J. Snelling, G. P. Flinn, A. S. Plaut, R. T. Harley, A. C. Tropper, R. Eccleston, and C. C. Phillips, Phys. Rev. B 44, 11345 (1991).

${ }^{15}$ A. A. Sirenko, T. Ruf, M. Cardona, D. R. Yakovlev, W. Ossau, A. Waag, and G. Landwehr, Phys. Rev. B 56, 2114 (1997).

${ }^{16}$ Wayne H. Lau, J. T. Olesberg, and Michael E. Flatte, Phys. Rev. B 64, 161301(R) (2001).

${ }^{17}$ M. W. Wu and C. Z. Ning, Eur. Phys. J. B 18, 373 (2000).

${ }^{18}$ J. Zhou and M. W. Wu, Phys. Rev. B 77, 075318 (2008); J. Zhou, J. L. Cheng, and M. W. Wu, ibid. 75, 045305 (2007).

${ }^{19}$ M. J. Yang, R. J. Wagner, B. V. Shanabrook, J. R. Waterman, and W. J. Moore, Phys. Rev. B 47, 6807 (1993).

${ }^{20}$ Zhigang Chen, Sam G. Carter, Rudolf Bratschitsch, Philip Dawson, and Steven T. Cundiff, Nat. Phys. 3, 265 (2007).

${ }^{21}$ This is only valid when $\tau_{p}$ is weak temperature dependent. We think it is acceptable for a rough estimation since $\tau_{p}$ in the submonolayer sample is mainly limited by the boundary scattering. 